Efecto del tamaño de las agujas en la inmunogenicidad y reactogenicidad de dos vacunas aplicadas a lactantes

Las agujas de inyección más gruesas y largas reducen significativamente la incidencia de reacciones locales en niños de 4 meses de edad. Sin embargo, no se ha determinado si ese efecto está relacionado con el largo o el calibre de las agujas. Tampoco se ha estudiado si las características de las agujas también influyen en la inmunogenicidad de las vacunas aplicadas a lactantes. Los beneficios derivados de la reducción de la reactogenicidad deben revalorarse en dependencia del efecto que tengan las agujas de inyección en la inmunogenicidad de las vacunas.

En este artículo se presentan los resultados de un ensayo aleatorizado controlado diseñado para comparar la reactogenicidad y la inmunogenicidad de una vacuna combinada tetravalente (contra la difteria, la tos ferina, el tétanos y Haemophilus influenzae tipo b) y de una vacuna conjugada contra meningococos del serogrupo $\mathrm{C}$ aplicadas con tres tipos diferentes de agujas: de $25 \mathrm{~mm}$ de largo y 23 gauges (G) de calibre, de $16 \mathrm{~mm}$ y $26 \mathrm{G}$ y de $25 \mathrm{~mm}$ y $25 \mathrm{G}$.

Se vacunaron 696 lactantes que recibieron su primera vacunación a los 2, 3 y 4 meses de edad entre abril de 2002 y marzo de 2004. Se excluyeron los niños que nacieron antes de cumplir 37 semanas de gestación, los que nacieron con menos de $2500 \mathrm{~g}$ de peso y los que presentaban alguna afección o tratamiento que pudieran influir en la respuesta inmunitaria. La primera vacunación se realizó a las 8-11 semanas de edad con dos revacunaciones con 4-6 semanas de intervalo. En el estudio participaron 18 de los 35 consultorios contactados en los consorcios de atención primaria de Vale of Aylesbury, Buckinghamshire y North East Oxfordshire, Reino Unido.

Las reacciones locales - tanto leves como graves - a la vacuna tetravalente disminuyeron significativamente cuando se emplearon agujas más largas y gruesas en comparación con las más finas y la más corta. Se observaron pequeñas diferencias en la reactogenicidad y la inmunogenicidad generadas por la vacunación con agujas del mismo largo pero de diferente calibre. La respuesta inmunitaria no disminuyó cuando se emplearon agujas de mayor longitud y calibre con cualquiera de las dos vacunas en comparación con la respuesta obtenida cuando se emplearon agujas más finas y la más corta.
No se encontraron diferencias significativas en la inmunogenicidad observada después de la vacunación con las agujas de $25 \mathrm{~cm}$ de largo, independientemente de su calibre. Las tasas de reacciones locales fueron ligeramente menores con la aguja más fina que con agujas más gruesas, pero esa diferencia fue pequeña y solamente fue estadísticamente significativa al analizar la tasa general de reacciones en la tercera dosis de la vacuna combinada y la tasa de reacciones de la tercera dosis a los 2 días de la vacunación. No se encontraron diferencias significativas en la reactogenicidad sistémica.

En conclusión, los resultados de este estudio indican que la vacunación de lactantes debe hacerse por vía intramuscular y que las agujas más largas favorecen la inmunogenicidad de la vacuna, independientemente de la técnica de inyección. Estos resultados se obtuvieron en una población de niños con buena alimentación, por lo que no se deben extrapolar acríticamente a otras poblaciones caracterizadas por un bajo estatus socioeconómico. Sin embargo, se debe dirigir la atención de los padres preocupados en reducir las reacciones locales hacia el interés por lograr una mayor eficacia de la vacuna y promover la utilización de agujas más largas. Tanto los fabricantes de vacunas como las autoridades nacionales deben tomar en cuenta el largo de las agujas en las recomendaciones que hacen para la vacunación infantil. (Diggle L, Deeks JJ, Pollard AJ. Effect of needle size on immunogenicity and reactogenicity of vaccines in infants: randomised controlled trial. Br Med J. 2006;333:571.)

\section{Confirman la gran diversidad de Leishmania en el estado de Acre, Brasil}

En Brasil, los parásitos del género Leishmania presentan una gran diversidad genética y se han descrito al menos siete especies: Leishmania (Viannia) braziliensis, Leishmania (Viannia) guyanensis, Leishmania (Viannia) shawi, Leishmania (Viannia) lainsoni, Leishmania (Viannia) naiffi, Leishmania (Viannia) lindenbergi y Leishmania (Leishmania) amazonensis. Todas ellas causan leishmaniasis en el hombre. Entre 1980 y 2003, las autoridades brasileñas documentaron más de medio millón de casos de leishmaniasis tegumentaria americana (LTA) en el país, con una incidencia anual que varió entre 3,83 y 22,94 casos por 100000 habitantes. Es importante identificar la especie de 
Leishmania causante de LTA no solo para propósitos taxonómicos y epidemiológicos, sino porque la eficacia de la respuesta a la vacuna pentavalente depende de la especie del parásito circulante.

El objetivo de este estudio fue describir las características clínicas, diagnósticas y epidemiológicas de la LTA en Acre, un estado de la Amazonia occidental brasileña, de acuerdo con la especie de Leishmania causante de la enfermedad.

El estudio se llevó a cabo entre marzo y septiembre de 2002 en el municipio de Rio Branco, donde se ha presentado $25 \%$ de los casos de LTA notificados por las autoridades sanitarias en los últimos 3 años. La muestra de conveniencia estuvo compuesta por 50 personas mayores de 6 años que presentaron lesiones cutáneas características de la leishmaniasis con más de dos semanas de evolución y sin afectación de las mucosas. Se emplearon técnicas convencionales y de biología molecular para confirmar el diagnóstico parasitológico e identificar la especie causante de la enfermedad.

Las características clínicas de la leishmaniasis cutánea observadas en este estudio fueron similares a las de otras regiones de Brasil, donde las especies pertenecientes al subgénero Leishmania (Viannia) son las responsables de la mayoría de los casos de LTA. El predominio de L. (V.) braziliensis y L. (V.) guyanensis en este estudio refleja la epidemiología de LTA en Brasil, ya que estas dos especies son las más frecuentemente descritas en las diferentes regiones endémicas del país. No se encontraron signos clínicos asociados con alguna especie en particular, lo que confirma el hecho de que es prácticamente imposible identificar la especie del parásito a partir de los signos y síntomas clínicos del paciente.

El creciente número de casos de LTA en el estado de Acre puede explicarse por el mejoramiento del sistema de vigilancia, las migraciones laborales de personas susceptibles hacia zonas de mayor transmisión, la afectación del equilibrio en los reservorios naturales debido a la deforestación y la explotación de los productos forestales, como frutas, caucho y madera.

Estos resultados muestran la gran diversidad del género Leishmania en el estado de Acre, donde circulan al menos tres especies y varios híbridos putativos. La diversidad de genotipos fue aun mayor, pues se describieron por primera vez nueve zimodemos, siete de ellos pertenecientes al complejo de $L$. $(V$.) braziliensis, lo que indica que hay una considerable diversidad aun dentro de una misma especie de este parásito.

Según estos resultados, se puede esperar que los ciclos de transmisión de este parásito sean también muy heterogéneos y que en ese ciclo participen varios vectores flebótomos y diversos organismos como reservorios. Por estas razones, las medidas de control basadas en el comportamiento del vector son sumamente complejas y evitar la transmisión puede ser muy difícil en regiones en las que hay políticas que respaldan la permanencia de personas susceptibles en zonas selváticas para realizar diversas actividades. (Tojal da Silva AC, Cupolillo E, Volpini AC, Almeida R, Sierra Romero GA. Species diversity causing human cutaneous leishmaniasis in Rio Branco, state of Acre, Brazil. Trop Med Int Health. 2006;11:1388-98.)

\section{Reflexiones sobre el desarrollo de capacidades para la promoción de salud en Perú}

En los últimos años, el desarrollo de capacidades ha estado en la agenda de los gobiernos donantes de recursos y de las agencias de desarrollo. El término "desarrollo de capacidades" se utiliza para denominar el proceso de creación y fortalecimiento de competencias y habilidades, tanto en instituciones como en personas individuales, que les facilite lograr el cumplimiento de sus objetivos.

En este artículo se analiza una intervención realizada en Perú para desarrollar capacidades dirigidas a la promoción de salud. La premisa básica de esa intervención fue que el fortalecimiento de la capacidad de las personas y de las instituciones para promover la salud contribuiría a mejorar las condiciones de salud y los hábitos saludables en todo el país.

A pesar de que en este trabajo no se hace un análisis final del impacto de esta intervención, ya que no se han evaluado formalmente las actividades realizadas, los resultados expuestos permiten extraer algunas lecciones que pueden ser de utilidad en el diseño de intervenciones dirigidas a desarrollar capacidades, especialmente para la promoción de salud.

La intervención se diseñó y llevó a cabo por el Proyecto Cambio entre 2001 y 2004 con la participación de cuatro universidades privadas (la Pontificia Universidad Católica, la Universidad Cayetano Heredia, la Universidad de Lima y la Universidad del Pacífico) y dos organizaciones no gubernamentales (ONG) peruanas con experiencia reconocida en la promoción de salud en la zona: Apoyo a Programas de Población (APROPO) y PRISMA. Se organizaron mesas redondas regionales en ocho regiones del país, talleres para los profesores, el personal de las ONG y el Ministerio de Salud de Perú, entrenamientos en la cobertura de noticias sobre salud para estudiantes y periodistas, un programa de becas dirigido a la comunicación y promoción de salud y un programa de prácticas estudiantiles en empresas.

Los resultados de esta intervención demuestran que el compromiso a largo plazo de los donantes $\mathrm{y}$ de las agencias de financiamiento es insuficiente 